\section{PSQ-088 DEVELOPMENT OF ALZHEIMER'S DISEASE AND EXPOSITION TO BENZODIAZEPINES: A COHORT STUDY IN A HEALTH REGION OF CATALONIA BETWEEN 2002 AND 2015}

${ }^{1}$ FI Torres-Bondia*, ${ }^{2} \mathrm{G}$ Piñol-Ripoll, ${ }^{3}$ De Batlle-Garcia, ${ }^{4} \mathrm{M}$ Buti-Sole, ${ }^{5} \mathrm{~L}$ Galvan-Santiago. ${ }^{1}$ Hospital Universitario Santa María de Lleida, Pharmacy, Lleida, Spain; ${ }^{2}$ Hospital Universitario Santa María de Lleida, Cognitive Disorders Unit, Lleida, Spain; ${ }^{3} / R B / L$ leida, Translational Research In Respiratory Medicine Research Group/lrblleida, Lleida, Spain; ${ }^{4}$ Institut Català de La Salut, Clinical Evaluation Unit, Lleida, Spain; ${ }^{5}$ Servei Català de La Salut, Pharmacy, Lleida, Spain

\subsection{6/ejhpharm-2019-eahpconf.521}

Background Alzheimer's disease (AD) is the main cause of dementia in developed countries. Sleep disturbances have been shown to increase the risk of $\mathrm{AD}$, however, benzodiazepine (BZD) consumption has also been shown to increase this risk in some cohort studies.

Purpose The objective of the study was to assess the risk of $\mathrm{AD}$ incidence in a cohort of patients exposed to BZD.

Material and methods Community-based retrospective cohort study from 1 January 2002 to 31 December 2015. Consumption was expressed in defined daily doses (DDD) accumulated by individuals. Three DDD intervals were established (1-90, 90-180 and >180). All approved BZD were included in the Medicines Catalogue of the Spanish Medicines Agency, as well as the BZD analogues (zoplicona, zolpidem). The patients treated with BZD during the 5 years immediately prior to diagnosis were excluded. The relationship between the BZD consumption categories and the development of $\mathrm{AD}$ was analysed by the $\mathrm{Chi}^{2}$ test and adjusted logistic regression models. Cox proportional hazards models were also used to consider the time to $\mathrm{AD}$ development.

Results The cohort included 84543 individuals consuming BZD and similar, with an average age in 2002 of 65 years. During follow-up, 584 new cases of AD were diagnosed. In the Cox models adjusted for year of birth, sex and comorbidities, taking as a reference the first category of BZD consumption (1-90 DDD), there was a 12-fold increase in the risk of developing $\mathrm{AD}$ in participants with cumulative consumption from 90 to 180 DDD (Hazard ratio (95\% CI): 11.6 (3.8-35.7), P-value $<0.001)$ and 78 times higher in participants with more than 180 accumulated DDD (Hazard ratio (95\% CI): $78.0 \quad(29.1-208.8), \quad P$-value<0.001). The study according to type of BZD revealed slightly higher incidences of $\mathrm{AD}$ in the participants in the highest category of consumption (>180 DDD) of BZD of intermediate-long action $1.20 \%$ with respect to those of short-intermediate action $1.11 \%$.

Conclusion The long-term use of BZD increases the risk of developing $\mathrm{AD}$. The establishment of new treatments with BZD should be restricted to the most serious cases and programmes of deprescription should be developed.

\section{REFERENCES AND/OR ACKNOWLEDGEMENTS}

https://www.bmj.com/content/352/bmj.i90

https://www.bmj.com/content/349/bmj.g5205

https://www.ncbi.nlm.nih.gov/pubmed/29273607

https://www.karger.com/Article/FullText/454881

No conflict of interest.

\section{PSQ-089 PREVALENCE OF PRESCRIPTION OF ANTIPSYCHOTIC POLYPHARMACY IN A PSYCHIATRIC HOSPITAL}

J Velasco*, M Martinez de Guzman, MA de Concepcion Salesa. Hospital Psiquiatrico Roman Alberca, Servicio de Farmacia, El Palmar, Spain

\subsection{6/ejhpharm-2019-eahpconf.522}

Background Despite a scanty evidence base and the number of adverse associations of antipsychotic combinations reported such as higher mortality, increased risk of side effects, adverse drug interactions, decreased treatment adherence and greater costs, the concurrent use of two or more antipsychotic medications continues to expand.

Purpose To analyse the prevalence of antipsychotic (APS) combination treatments in patients admitted to the Acute and Half-Stay Units, as well as its concomitance with antidepressants and mood stabilisers.

Material and methods Descriptive cross-sectional study.

Information collected: history, sex, age, diagnosis, antipsychotics, antidepressants and mood stabilisers.

Statistical analysis with SPSS, significance level 0.05.

Results Eighty-two patients $(56.1 \%$ males, $43.9 \%$ females; mean age of $42.7 \pm 11.3$ years, $51.2 \%$ with schizophrenia (F20), 19.5\% schizoaffective disorder (F25), 14.6\% with personality disorder (F60), 7.3\% with bipolar disorder (F31) and $7.3 \%$ with other diagnoses (according to DSM-IV, ICD10). $23.2 \%$ were in the Short-Stay Unit and the rest (76.8\%) in the Half-Stay Unit. Substance addiction was present in $42.7 \%$.

APS per patient was $1.8 \pm 0.8$, of which $0.2 \pm 0.4$ were typical and $1.6 \pm 0.7$ atypical. Number of prescriptions with antipsychotics were 80 (97.6\%), of which three (3.7\%) contained only typical, $64(78.0 \%)$ atypical and 13 (15.9\%) both APS $\left(\mathrm{Chi}^{2}=126.7 ; \mathrm{p}<0.01\right)$. Thirty-four $(42.5 \%)$ were prescribed long-acting injectable antipsychotics.

$45.1 \%(0.6 \pm 0.8$ per patient $)$ were prescribed antidepressants, $36.6 \%(0.5 \pm 0.7) \operatorname{mood}$ stabilisers and $91.5 \%(1.7 \pm 0.9)$ benzodiazepines.

$32.9 \% \quad(\mathrm{n}=27)$ received antipsychotic monotherapy, two APS 51.2\% ( $n=42 \%)$, three APS 11.0\% $(n=9)$ and four APS $2.4 \%(\mathrm{n}=2)\left(\mathrm{Chi}^{2}=73.4 ; \mathrm{p}<0.01\right)$. The doses were exceeded in six $(7.3 \%)$ prescriptions according to the relevant Summary of Product Characteristics for these medicinal products.

The prevalence of typical antipsychotics was: clotiapine eight (9.8\%), levomepromazine three (3.7\%), zuclopenthixol three $(3.7 \%)$ and haloperidol two (2.4\%). The prevalence of atypical APS was: clozapine 13 (15.9\%), olanzapine 17 (20.7\%), quetiapine $2226.8 \%)$, paliperidone 20 (24.4\%), risperidone $25(30.5 \%)$, aripiprazole 25 (30.5\%), ziprasidone six (7.3\%) and amisulpiride four (4.9\%).

Conclusion The high use of antipsychotics (67.1\%) which, although it may reflect the complexity and resistance of the treated pathologies, does not agree with the recommendations of the national and international guidelines.

Combining mood stabilisers with atypical antipsychotics or antidepressants when resistant pathology can result in therapeutic synergy and obtain a more favourable result. This combination can accelerate the response to treatment and minimise adverse effects by allowing the use of lower doses of each drug. The particular characteristics of each patient must be considered. 